\title{
CONJUGACY SEPARABILITY OF CERTAIN GENERALIZED FREE PRODUCTS OF NILPOTENT GROUPS
}

\author{
Goansu Kim and C. Y. TAng
}

\begin{abstract}
It is known that generalized free products of finitely generated nilpotent groups are conjugacy separable when the amalgamated subgroups are cyclic or central in both factor groups. However, those generalized free products may not be conjugacy separable when the amalgamated subgroup is a direct product of two infinite cycles. In this paper we show that generalized free products of finitely generated nilpotent groups are conjugacy separable when the amalgamated subgroup is $\langle h\rangle \times D$, where $D$ is in the center of both factors.
\end{abstract}

\section{Introduction}

Let $S$ be a subset of a group $G$. Then $G$ is said to be $S$-separable if for each $x \in G \backslash S$, there exists a normal subgroup $N_{x}$ of finite index in $G$ such that $x \notin N_{x} S$. Equivalently, $S$ is a closed subset of $G$ in the profinite topology of $G$. If $S=\{1\}$, then $G$ is residually finite $(\mathcal{R} F)$. If for each $x \in G, G$ is $\{x\}^{G}$-separable, where $\{x\}^{G}$ is the conjugacy class of $x$ in $G$, then $G$ is called conjugacy separable (c.s.). Residual and separability properties are of interest to both group theorists and topologists. They are related to the solvability of the word problem, the conjugacy problem and the generalized word problem (Mal'cev [11] and Mostowski [12]). Topologically, they are related to problems on the embeddability of equivariant subspaces in their regular covering spaces (Scott [18], Niblo [13]).

Blackburn [2] first proved that finitely generated nilpotent groups are conjugacy separable. Stebe showed that free products of conjugacy separable groups are conjugacy separable [19], hence free groups are conjugacy separable. Formanek [6] (also Remeslennikov [14]) showed that polycyclic-by-finite groups

Received July 4, 2011; Revised June 19, 2012.

2010 Mathematics Subject Classification. Primary 20E26, 20E06; Secondary 20F10.

Key words and phrases. generalized free products, residually finite, conjugacy separable, nilpotent groups.

The first author was supported by Basic Science Research Program though the NRF funded by the Ministry of Education, Science and Technology (2012R1A1A2002595).

The second author gratefully acknowledges the partial support by the Natural Science and Engineering Research Council of Canada, Grant No. A-4064. 
are also conjugacy separable. Dyer [3] showed that free-by-finite groups are conjugacy separable. There are a number of other results on conjugacy separability. In particular, Fine and Rosenberger [5] proved that Fuchsian groups are conjugacy separable. Wilson and Zalesskii [21] showed that the Bianchi groups $\operatorname{PSL}_{2}\left(\mathcal{O}_{d}\right)$, where $\mathcal{O}_{d}$ is the ring of integers of $\mathbb{Q}(\sqrt{-d})$ for $d=1,2,7,11$, are conjugacy separable.

In general, the generalized free product of two conjugacy separable groups is not conjugacy separable. Baumslag [1] constructed an example of a generalized free product of two finitely generated nilpotent groups amalgamating a direct product of two cycles (see Example 2.1 below) which is not even Hopfian, whence not conjugacy separable. However, Dyer [4] showed that the free product of two free groups - or two finitely generated nilpotent groups- amalgamating a cyclic subgroup is conjugacy separable. Tang [20] showed that generalized free products of surface groups amalgamating a cyclic subgroup are conjugacy separable. Ribes, Segal and Zalesskii [16] showed that generalized free products of polycyclic groups amalgamating cyclic subgroups are conjugacy separable.

As mentioned above, generalized free products of finitely generated nilpotent groups amalgamating a cyclic subgroup are conjugacy separable. But those generalized free products amalgamating a direct product of two cyclic subgroups may not be conjugacy separable. However, those generalized free products amalgamating a central subgroup are conjugacy separable [8]. In this paper, we find some conditions to derive that generalized free products of conjugacy separable groups amalgamating $\langle h\rangle \times D$, where $D$ is in the center of both factors, are conjugacy separable. Using this, we show that generalized free products of finitely generated nilpotent groups amalgamating $\langle h\rangle \times D$, where $D$ is in the center of both factors, are conjugacy separable.

\section{Preliminaries}

Throughout this paper we use standard notation and terminology.

If $A, B$ are groups, $G=A *_{H} B$ denotes the generalized free product of $A$ and $B$ amalgamating the subgroup $H$. If $x \in G=A *_{H} B$, then $\|x\|$ denotes the free product length of $x$ in $G$.

$x \sim_{G} y$ means that $x$ and $y$ are conjugate in $G$.

$Z(G)$ is the center of $G$.

$C_{H}(u)=\{h \in H \mid h u=u h\}$ denotes the centralizer of $u$ in $H$.

$\mathcal{R} F$ is an abbreviation for "residually finite".

The following example shows that the generalized free product of two finitely generated free nilpotent groups amalgamating a subgroup isomorphic to $\mathbb{Z} \times \mathbb{Z}$ may not be residually finite.

Example 2.1 ([1]). Let $A=\langle a, b ;[a, b, b]=[a, b, a]=1\rangle$ and $B=\langle c, d ;[c, d, d]$ $=[c, d, c]=1\rangle$. Let $H=\left\langle a,\left[a^{2}, b\right]\right\rangle$ and $K=\langle[c, d], c\rangle$. Clearly, $A, B$ are free-nilpotent groups of class $2,\left[a^{2}, b\right] \in Z(A)$, and $[c, d] \in Z(B)$. Let $G$ be 
the generalized free product of $A$ and $B$ amalgamating $H$ and $K$ by letting $a=[c, d]$ and $\left[a^{2}, b\right]=c$. Then $G$ is non-Hopfian, whence $G$ is not conjugacy separable.

Theorem 2.2 ([10, Theorem 4.6]). Let $G=A *_{H} B$ and let $x \in G$ be of minimal length in its conjugacy class. Suppose that $y \in G$ is cyclically reduced and that $x \sim_{G} y$.

(1) If $\|x\|=0$, then $\|y\| \leq 1$ and if $y \in A$, then there is a sequence $h_{1}, h_{2}, \ldots, h_{r}$ of elements in $H$ such that $y \sim_{A} h_{1} \sim_{B} h_{2} \sim_{A} \cdots \sim_{A(B)} h_{r}=x$.

(2) If $\|x\|=1$, then $\|y\|=1$ and either $x, y \in A$ and $x \sim_{A} y$ or $x, y \in B$ and $x \sim_{B} y$.

(3) If $\|x\| \geq 2$, then $\|x\|=\|y\|$ and $y \sim_{H} x^{*}$, where $x^{*}$ is a cyclic permutation of $x$.

\section{Double coset separability}

In this section we prove some results on double coset separability.

Definition 3.1. Let $G$ be a group and $H, K$ be subgroups of $G$. Then $G$ is said to be $H K$-double coset separable if for each $x \in G, G$ is $H x K$-separable. In particular, we say that $G$ is $H$-double coset separable if $G$ is $H x H$-separable for all $x \in G$.

Clearly, if $G$ is $H$-double coset separable, then $G$ is $H$-separable. In particular, free groups [17] and finitely generated nilpotent groups [9] are $H$-double coset separable for each finitely generated subgroup $H$.

Lemma 3.2. Let $A$ be a group and $h \in A$ with $|h|=\infty$. Suppose $a \in A$ and $a \neq h^{i} a h^{j}$ for all integers $i, j$ except $i=j=0$. If $A$ is $\left\langle h^{n}\right\rangle$-double coset separable, then there exists $M \triangleleft_{f} A$ such that in $\bar{A}=A / M$, if $\bar{a}=\bar{h}^{i} \bar{a}^{j}$, then $n \mid i, j$.

Proof. We note that for $0 \leq i_{1}, j_{1} \leq n-1$, if $h^{-i_{1}} a h^{-j_{1}} \in\left\langle h^{n}\right\rangle a\left\langle h^{n}\right\rangle$, then $i_{1}=j_{1}=0$. Hence $h^{-i_{1}} a h^{-j_{1}} \notin\left\langle h^{n}\right\rangle a\left\langle h^{n}\right\rangle$ for all $0 \leq i_{1}, j_{1} \leq n-1$ except $i_{1}=j_{1}=0$. Since $A$ is $\left\langle h^{n}\right\rangle$-double coset separable, there exists $M \triangleleft_{f} A$ such that $h^{-i_{1}} a h^{-j_{1}} \notin M\left\langle h^{n}\right\rangle a\left\langle h^{n}\right\rangle$ for all $0 \leq i_{1}, j_{1} \leq n-1$ except $i_{1}=j_{1}=0$. Then in $\bar{A}=A / M$, if $\bar{a}=\bar{h}^{i} \bar{a}^{j}$, then $n \mid i, j$.

Definition 3.3. Let $G$ be a group and $h \in G$. Then $G$ is called $\langle h\rangle$-selfconjugate if $h^{i} \sim_{G} h^{j}$ implies $i=j$.

For example, free groups and finitely generated nilpotent groups are $\langle h\rangle$-selfconjugate for each element $h$ of infinite order (see [4]).

Definition 3.4. Let $G$ be a group and $h \in G$ with $|h|=\infty$. Then we say that $G$ satisfies $\left(C^{\prime}\right)$ for $\langle h\rangle$ if there exists an integer $\delta>0$ such that for each $n>0$, there exists $M \triangleleft_{f} G$ depending on $n$ such that $M \cap\langle h\rangle=\left\langle h^{n \delta}\right\rangle$ and if $M h^{i} \sim_{G / M} M h^{j}$, then $M h^{i}=M h^{j}$. 
Theorem 3.5. Let $G=A *\langle h\rangle B$, where $|h|=\infty$. Let $A, B$ be $\langle h\rangle$-self-conjugate and $\left\langle h^{n}\right\rangle$-double coset separable for each integer $n>0$. Suppose $A, B$ satisfy the condition $\left(C^{\prime}\right)$ for $\langle h\rangle$. Then $G$ is $\langle h\rangle$-double coset separable.

Proof. Let $x, y \in G$ and $y \notin\langle h\rangle x\langle h\rangle$.

Case 1. $\|x\| \neq\|y\|$. Suppose $x=a_{1} b_{1} \cdots a_{n} b_{n}$ and $y=c_{1} d_{1} \cdots c_{m} d_{m}$, where $a_{i}, c_{j} \in A \backslash\langle h\rangle$ and $b_{i}, d_{j} \in B \backslash\langle h\rangle$. Since $A, B$ are $\langle h\rangle$-double coset separable, $A, B$ are $\langle h\rangle$-separable. Hence there exist $M_{1} \triangleleft_{f} A$ and $N_{1} \triangleleft_{f} B$ such that $a_{i}, c_{j} \notin M_{1}\langle h\rangle$ and $b_{i}, d_{j} \notin N_{1}\langle h\rangle$ for all $i, j$. Let $M_{1} \cap\langle h\rangle=\left\langle h^{s_{1}}\right\rangle$ and $N_{1} \cap\langle h\rangle=\left\langle h^{s_{2}}\right\rangle$ for some integers $s_{1}, s_{2}$. By $\left(C^{\prime}\right)$, there exists an integer $\delta_{1}>0$ such that for each $n>0$, there exists $M^{\prime} \triangleleft_{f} A$ such that $M^{\prime} \cap\langle h\rangle=\left\langle h^{n \delta_{1}}\right\rangle$ and if $M^{\prime} h^{i} \sim_{A / M^{\prime}} M^{\prime} h^{j}$, then $M^{\prime} h^{i}=M^{\prime} h^{j}$. Similarly, there exists an integer $\delta_{2}>0$ such that for each $n>0$, there exists $N^{\prime} \triangleleft_{f} B$ such that $N^{\prime} \cap\langle h\rangle=\left\langle h^{n \delta_{2}}\right\rangle$ and if $N^{\prime} h^{i} \sim_{B / N^{\prime}} N^{\prime} h^{j}$, then $N^{\prime} h^{i}=N^{\prime} h^{j}$. Hence considering $n=s_{1} s_{2} \delta_{2}$, there exists $M_{2} \triangleleft_{f} A$ such that $M_{2} \cap\langle h\rangle=\left\langle h^{s_{1} s_{2} \delta_{1} \delta_{2}}\right\rangle$ and if $M_{2} h^{i} \sim_{A / M_{2}} M_{2} h^{j}$, then $M_{2} h^{i}=M_{2} h^{j}$. Similarly, there exists $N_{2} \triangleleft_{f} B$ such that $N_{2} \cap\langle h\rangle=\left\langle h^{s_{1} s_{2} \delta_{1} \delta_{2}}\right\rangle$ and if $N_{2} h^{i} \sim_{B / N_{2}} N_{2} h^{j}$, then $N_{2} h^{i}=N_{2} h^{j}$. Let $M=M_{1} \cap M_{2}$ and $N=$ $N_{1} \cap N_{2}$. Clearly, $M \cap\langle h\rangle=\left\langle h^{s_{1} s_{2} \delta_{1} \delta_{2}}\right\rangle=N \cap\langle h\rangle$. Then in $\bar{G}=\bar{A} *\langle\bar{h}\rangle \bar{B}$, where $\bar{A}=A / M$ and $\bar{B}=B / N$, we have $\|\bar{x}\|=\|x\|$ and $\|\bar{y}\|=\|y\|$. Since $\|x\| \neq\|y\|,\|\bar{x}\| \neq\|\bar{y}\|$. This implies that $\bar{y} \notin\langle\bar{h}\rangle \bar{x}\langle\bar{h}\rangle$. Since $\bar{G}$ is free-by-finite, it is residually finite. We can find $\bar{P} \triangleleft_{f} \bar{G}$ such that $\bar{y} \notin \bar{P}\langle\bar{h}\rangle \bar{x}\langle\bar{h}\rangle$. Let $P$ be the preimage of $\bar{P}$ in $G$. Then $P \triangleleft_{f} G$ and $y \notin P\langle h\rangle x\langle h\rangle$.

The case when $\|x\|=0$ and $\|y\| \neq 0$ (or $\|x\| \neq 0$ and $\|y\|=0$ ) also can be similarly considered.

Case 2. $\|x\|=\|y\| \leq 1$. If $x \in A \backslash\langle h\rangle$ and $y \in B \backslash\langle h\rangle$, then the above method can be applied. So we suppose $x, y \in A$. Since $A$ is $\langle h\rangle$-double coset separable, there exists $M_{1} \triangleleft_{f} A$ such that $y \notin M_{1}\langle h\rangle x\langle h\rangle$. Let $M_{1} \cap\langle h\rangle=$ $\left\langle h^{s_{1}}\right\rangle$ for some integer $s_{1}$. By $\left(C^{\prime}\right)$, there exist $M_{2} \triangleleft_{f} A$ and $N_{2} \triangleleft_{f} B$ such that $M_{2} \cap\langle h\rangle=\left\langle h^{s_{1} \delta_{1} \delta_{2}}\right\rangle=N_{2} \cap\langle h\rangle$. Let $M=M_{1} \cap M_{2}$ and $N=N_{2}$. Then in $\bar{G}=A / M *_{\langle\bar{h}\rangle} B / N, \bar{y} \notin\langle\bar{h}\rangle \bar{x}\langle\bar{h}\rangle$. Hence, as before, we can find $\bar{P} \triangleleft_{f} \bar{G}$ such that $\bar{y} \notin \bar{P}\langle\bar{h}\rangle \bar{x}\langle\bar{h}\rangle$. Let $P$ be the preimage of $\bar{P}$ in $G$. Then $P \triangleleft_{f} G$ and $y \notin P\langle h\rangle x\langle h\rangle$.

Case 3. $\|x\|=\|y\| \geq 2$. Suppose $x=a_{1} b_{1} \cdots a_{n} b_{n}$ and $y=d_{1} c_{1} \cdots d_{n} c_{n}$, where $a_{i}, c_{i} \in A \backslash\langle h\rangle$ and $b_{i}, d_{j} \in B \backslash\langle h\rangle$. This case can be similarly handled as in Case 1.

Suppose $x=a_{1} b_{1} \cdots a_{n} b_{n}$ and $y=c_{1} d_{1} \cdots c_{n} d_{n}$, where $a_{i}, c_{i} \in A \backslash\langle h\rangle$ and $b_{i}, d_{i} \in B \backslash\langle h\rangle$.

(1) Suppose there exists $i$ such that $c_{i} \notin\langle h\rangle a_{i}\langle h\rangle$ (or $d_{i} \notin\langle h\rangle b_{i}\langle h\rangle$ ). As in Case 1 above, we can find $M \triangleleft_{f} A$ and $N \triangleleft_{f} B$ such that in $\bar{G}=A / M *_{\langle\bar{h}\rangle} B / N$, $\bar{c}_{i} \notin\langle\bar{h}\rangle \bar{a}_{i}\langle\bar{h}\rangle,\|\bar{x}\|=\|x\|$ and $\|\bar{y}\|=\|y\|$. Then $\bar{y} \notin\langle\bar{h}\rangle \bar{x}\langle\bar{h}\rangle$. For, if $\bar{y} \in\langle\bar{h}\rangle \bar{x}\langle\bar{h}\rangle$, then there exist integers $\alpha_{i}, \mu_{i}$ such that

$$
\bar{c}_{1}=\bar{h}^{-\alpha_{1}} \bar{a}_{1} \bar{h}^{\mu_{1}}
$$




$$
\begin{gathered}
\bar{d}_{1}=\bar{h}^{-\mu_{1}} \bar{b}_{1} \bar{h}^{\alpha_{2}} \\
\vdots \\
\bar{c}_{i}=\bar{h}^{-\alpha_{i}} \bar{a}_{i} \bar{h}^{\mu_{i}} \\
\vdots \\
\bar{d}_{n}=\bar{h}^{-\mu_{n}} \bar{b}_{n} \bar{h}^{\alpha_{n}} .
\end{gathered}
$$

Hence $\bar{c}_{i} \in\langle\bar{h}\rangle \bar{a}_{i}\langle\bar{h}\rangle$, a contradiction. Therefore, $\bar{y} \notin\langle\bar{h}\rangle \bar{x}\langle\bar{h}\rangle$. As before, we can find $P \triangleleft_{f} G$ such that $y \notin P\langle h\rangle x\langle h\rangle$.

(2) Suppose $c_{k} \in\langle h\rangle a_{k}\langle h\rangle$ and $d_{k} \in\langle h\rangle b_{k}\langle h\rangle$ for all $k$. Then there exists $i$ such that $c_{1} d_{1} \cdots c_{i} \in\langle h\rangle a_{1} b_{1} \cdots a_{i}\langle h\rangle$ and $c_{1} d_{1} \cdots c_{i} d_{i} \notin\langle h\rangle a_{1} b_{1} \cdots a_{i} b_{i}\langle h\rangle$ (or, similarly, $c_{1} d_{1} \cdots d_{i} \in\langle h\rangle a_{1} b_{1} \cdots b_{i}\langle h\rangle$ and $c_{1} d_{1} \cdots d_{i} c_{i+1} \notin\langle h\rangle a_{1} b_{1} \cdots$ $\left.b_{i} a_{i+1}\langle h\rangle\right)$. Let $c_{1} d_{1} \cdots c_{i}=h^{\lambda_{1}} a_{1} b_{1} \cdots a_{i} h^{\lambda_{2}}$ and $d_{i}=h^{\beta_{1}} b_{i} h^{\beta_{2}}$ for some integers $\lambda_{1}, \lambda_{2}, \beta_{1}, \beta_{2}$. Hence $a_{1} b_{1} \cdots a_{i} h^{\lambda_{2}+\beta_{1}} b_{i} \notin\langle h\rangle a_{1} b_{1} \cdots a_{i} b_{i}\langle h\rangle$. For convenience, let $w=a_{1} b_{1} \cdots a_{i}$. Then $w h^{\lambda_{2}+\beta_{1}} b_{i} \notin\langle h\rangle w b_{i}\langle h\rangle$. Hence $h^{\lambda_{2}+\beta_{1}} \notin$ $C_{\langle h\rangle}(w) \cdot C_{\langle h\rangle}\left(b_{i}\right)$. Let $C_{\langle h\rangle}(w)=\left\langle h^{s}\right\rangle$ and $C_{\langle h\rangle}\left(b_{i}\right)=\left\langle h^{t}\right\rangle$. Then $h^{\lambda_{2}+\beta_{1}} \notin$ $\left\langle h^{s}\right\rangle\left\langle h^{t}\right\rangle$.

(a) $s=0$ and $t \neq 0$ (or $s \neq 0$ and $t=0$ ). Clearly, $h^{\lambda_{2}+\beta_{1}} \notin\left\langle h^{t}\right\rangle$. Since $A$ is $\langle h\rangle$-self-conjugate and $C_{\langle h\rangle}\left(b_{i}\right)=\left\langle h^{t}\right\rangle$, we have $b_{i}^{-1} h^{j_{1}} b_{i} \notin\langle h\rangle$ for all $0 \leq j_{1}<t$. Since $C_{\langle h\rangle}(w)=1$, there exists $r$ such that $C_{\langle h\rangle}\left(a_{r}\right)=1$ for some $1 \leq r \leq i$ (or similarly, $C_{\langle h\rangle}\left(b_{r}\right)=1$ for some $1 \leq r<i$ ). This implies that $a_{r} \neq h^{j} a_{r} h^{j^{\prime}}$ for all $j, j^{\prime}$ except $j=j^{\prime}=0$. Since $A$ is $\left\langle h^{n}\right\rangle$-double coset separable for each $n>0, A$ is $\left\langle h^{n}\right\rangle$-separable for each $n>0$. Hence there exists $M_{1} \triangleleft_{f} A$ such that $h^{\lambda_{2}+\beta_{1}} \notin M_{1}\left\langle h^{t}\right\rangle, a_{k} \notin M_{1}\langle h\rangle$ for all $1 \leq k \leq n$ and if $M_{1} a_{r}=M_{1} h^{j} a_{r} h^{j^{\prime}}$, then $t \mid j, j^{\prime}$ (Lemma 3.2). Since $B$ is $\langle h\rangle$-self-conjugate and $C_{\langle h\rangle}\left(b_{i}\right)=\left\langle h^{t}\right\rangle$, we have $b_{i}^{-1} h^{j_{1}} b_{i} \notin\langle h\rangle$ for all $0 \leq j_{1}<t$. Hence there exists $N_{1} \triangleleft_{f} B$ such that $b_{k} \notin N_{1}\langle h\rangle$ for all $1 \leq k \leq n$ and $b_{i}^{-1} h^{j_{1}} b_{i} \notin N_{1}\langle h\rangle$ for all $0 \leq j_{1}<t$. Let $M_{1} \cap\langle h\rangle=\left\langle h^{s_{1}}\right\rangle$ and $N_{1} \cap\langle h\rangle=\left\langle h^{s_{2}}\right\rangle$ for some integers $s_{1}, s_{2}$. By $\left(C^{\prime}\right)$, there exists $M_{2} \triangleleft_{f} A$ such that $M_{2} \cap\langle h\rangle=\left\langle h^{s_{1} s_{2} \delta_{1} \delta_{2}}\right\rangle$ and if $M_{2} h^{j} \sim_{A / M_{2}} M_{2} h^{j^{\prime}}$, then $M_{2} h^{j}=M_{2} h^{j^{\prime}}$. Similarly, there exists $N_{2} \triangleleft_{f} B$ such that $N_{2} \cap\langle h\rangle=\left\langle h^{s_{1} s_{2} \delta_{1} \delta_{2}}\right\rangle$, and if $N_{2} h^{j} \sim_{B / N_{2}} N_{2} h^{j^{\prime}}$, then $N_{2} h^{j}=N_{2} h^{j^{\prime}}$. Let $M=M_{1} \cap M_{2}$ and $N=N_{1} \cap N_{2}$. Then in $\bar{G}=A / M *\langle\bar{h}\rangle / N$, we have $\|\bar{x}\|=\|x\|$ and $\bar{w} \bar{h}^{\lambda_{2}+\beta_{1}} \bar{b}_{i} \notin\langle\bar{h}\rangle \bar{w} \bar{b}_{i}\langle\bar{h}\rangle$. For, if $\bar{w} \bar{h}^{\lambda_{2}+\beta_{1}} \bar{b}_{i} \in\langle\bar{h}\rangle \bar{w} \bar{b}_{i}\langle\bar{h}\rangle$, then there exist integers $\alpha_{i}, \mu_{i}$ such that

$$
\begin{gathered}
\bar{a}_{1}=\bar{h}^{-\alpha_{1}} \bar{a}_{1} \bar{h}^{\mu_{1}} \\
\bar{b}_{1}=\bar{h}^{-\mu_{1}} \bar{b}_{1} \bar{h}^{\alpha_{2}} \\
\vdots \\
\bar{a}_{r}=\bar{h}^{-\alpha_{r}} \bar{a}_{r} \bar{h}^{\mu_{r}}
\end{gathered}
$$




$$
\begin{aligned}
\bar{a}_{i} & =\bar{h}^{-\alpha_{i}} \bar{a}_{i} \bar{h}^{\mu_{i}} \\
\bar{h}^{\lambda_{2}+\beta_{1}} \bar{b}_{i} & =\bar{h}^{-\mu_{i}} \bar{b}_{i} \bar{h}^{\alpha_{i}} .
\end{aligned}
$$

From the first equation in (3.1), we have $\bar{h}^{\alpha_{1}} \sim_{\bar{A}} \bar{h}^{\mu_{1}}$. By the choice of $M_{2}$, $M_{2} h^{\alpha_{1}}=M_{2} h^{\mu_{1}}$. Hence $h^{\alpha_{1}-\mu_{1}} \in M_{2} \cap\langle h\rangle \subset M_{1} \cap\langle h\rangle$. Thus $h^{\alpha_{1}-\mu_{1}} \in$ $M_{1} \cap M_{2}=M$, whence $\bar{h}^{\alpha_{1}}=\bar{h}^{\mu_{1}}$. Similarly, $\bar{h}^{\mu_{1}}=\bar{h}^{\alpha_{2}}, \ldots, \bar{h}^{\alpha_{i}}=\bar{h}^{\mu_{i}}$. Hence, $\bar{h}^{\alpha_{1}}=\bar{h}^{\mu_{1}}=\cdots=\bar{h}^{\mu_{i}}$. By the choice of $r$, we have $t \mid \alpha_{r}, \mu_{r}$. Hence $\bar{h}^{\mu_{i}}=\bar{h}^{\mu_{r}} \in\left\langle\bar{h}^{t}\right\rangle$. From the last equation of (3.1), we have $\bar{b}_{i}^{-1} \bar{h}^{\lambda_{2}+\beta_{1}+\mu_{i}} \bar{b}_{i}=$ $\bar{h}^{\alpha_{i}}$. By the choice of $N_{1}, t \mid \lambda_{2}+\beta_{1}+\mu_{i}$. Hence $\bar{h}^{\lambda_{2}+\beta_{1}+\mu_{i}}=\bar{h}^{\alpha_{i}} \in\left\langle\bar{h}^{t}\right\rangle$. Thus $\bar{h}^{\lambda_{2}+\beta_{1}}=\bar{h}^{\alpha_{i}-\mu_{i}} \in\left\langle\bar{h}^{t}\right\rangle$, a contradiction by the choice of $M_{1}$. Thus $\bar{w} \bar{h}^{\lambda_{2}+\beta_{1}} \bar{b}_{i} \notin\langle\bar{h}\rangle \bar{w} \bar{b}_{i}\langle\bar{h}\rangle$, and hence $\bar{c}_{1} \bar{d}_{1} \cdots \bar{c}_{i} \bar{d}_{i} \notin\langle\bar{h}\rangle \bar{a}_{1} \bar{b}_{1} \cdots \bar{a}_{i} \bar{b}_{i}\langle\bar{h}\rangle$. Therefore, $\bar{y} \notin\langle\bar{h}\rangle \bar{x}\langle\bar{h}\rangle$. Since $\bar{G}=A / M *\langle\bar{h}\rangle=B / N$ is residually finite, we can find $P \triangleleft_{f} G$ such that $y \notin P\langle h\rangle x\langle h\rangle$.

(b) $s=0$ and $t=0$. Clearly, $h^{\lambda_{2}+\beta_{1}} \neq 1$. Since $C_{\langle h\rangle}(w)=1$, there exists $r$ such that $C_{\langle h\rangle}\left(a_{r}\right)=1$ for some $1 \leq r \leq i$ (or $C_{\langle h\rangle}\left(b_{r}\right)=1$ for some $1 \leq r<i)$. Clearly, $h^{\lambda_{2}+\beta_{1}} \notin h^{2\left(\lambda_{2}+\beta_{1}\right)}$. As in (a) above, $a_{r} \neq h^{j} a_{r} h^{j^{\prime}}$ for all $j, j^{\prime}$ except $j=j^{\prime}=0$. Since $A$ is $\left\langle h^{n}\right\rangle$-double coset separable, $A$ is $\left\langle h^{2\left(\lambda_{2}+\beta_{1}\right)}\right\rangle$-separable and $\langle h\rangle$-separable. Hence there exists $M_{1} \triangleleft_{f} A$ such that $h^{\lambda_{2}+\beta_{1}} \notin M_{1}\left\langle h^{2\left(\lambda_{2}+\beta_{1}\right)}\right\rangle, a_{k} \notin M_{1}\langle h\rangle$ for all $1 \leq k \leq n$ and if $M_{1} a_{r}=$ $M_{1} h^{j} a_{r} h^{j^{\prime}}$, then $2\left(\lambda_{2}+\beta_{1}\right) \mid j, j^{\prime}$ (Lemma 3.2). Now, since $t=0, C_{\langle h\rangle}\left(b_{i}\right)=1$. Hence $b_{i} \neq h^{j} b_{r} h^{j^{\prime}}$ for all $j, j^{\prime}$ except $j=j^{\prime}=0$. As before, there exists $N_{1} \triangleleft_{f} B$ such that $b_{k} \notin N_{1}\langle h\rangle$ for all $1 \leq k \leq n$ and $N_{1} b_{i}=N_{1} h^{j} b_{i} h^{j^{\prime}}$. Then $2\left(\lambda_{2}+\beta_{1}\right) \mid j, j^{\prime}$. Let $M_{1} \cap\langle h\rangle=\left\langle h^{s_{1}}\right\rangle$ and $N_{1} \cap\langle h\rangle=\left\langle h^{s_{2}}\right\rangle$ for some integers $s_{1}, s_{2}$. By $\left(C^{\prime}\right)$, there exists $M_{2} \triangleleft_{f} A$ such that $M_{2} \cap\langle h\rangle=\left\langle h^{s_{1} s_{2} \delta_{1} \delta_{2}}\right\rangle$ and if $M_{2} h^{j} \sim_{A / M_{2}} M_{2} h^{j^{\prime}}$, then $M_{2} h^{j}=M_{2} h^{j^{\prime}}$. Similarly, there exists $N_{2} \triangleleft_{f} B$ such that $N_{2} \cap\langle h\rangle=\left\langle h^{s_{1} s_{2} \delta_{1} \delta_{2}}\right\rangle$, and if $N_{2} h^{j} \sim_{B / N_{2}} N_{2} h^{j^{\prime}}$, then $N_{2} h^{j}=N_{2} h^{j^{\prime}}$. Let $M=M_{1} \cap M_{2}$ and $N=N_{1} \cap N_{2}$. Then in $\bar{G}=A / M *\langle\bar{h}\rangle B / N$, we have $\|\bar{x}\|=\|x\|$ and $\bar{w} \bar{h}^{\lambda_{2}+\beta_{1}} \bar{b}_{i} \notin\langle\bar{h}\rangle \bar{w} \bar{b}_{i}\langle\bar{h}\rangle$. For, if $\bar{w} \bar{h}^{\lambda_{2}+\beta_{1}} \bar{b}_{i} \in\langle\bar{h}\rangle \bar{w} \bar{b}_{i}\langle\bar{h}\rangle$, then there exist integers $\alpha_{i}, \mu_{i}$ such that

$$
\begin{aligned}
\bar{a}_{1} & =\bar{h}^{-\alpha_{1}} \bar{a}_{1} \bar{h}^{\mu_{1}} \\
\bar{b}_{1} & =\bar{h}^{-\mu_{1}} \bar{b}_{1} \bar{h}^{\alpha_{2}} \\
\vdots & \\
\bar{a}_{r} & =\bar{h}^{-\alpha_{r}} \bar{a}_{r} \bar{h}^{\mu_{r}} \\
\vdots & \\
\bar{h}^{\lambda_{2}+\beta_{1}} \bar{b}_{i} & =\bar{h}^{-\mu_{i}} \bar{b}_{i} \bar{h}^{\alpha_{i}} .
\end{aligned}
$$


As in (a) above, from the equations in (3.2), we have $\bar{h}^{\alpha_{1}}=\bar{h}^{\mu_{1}}=\cdots=\bar{h}^{\mu_{i}}$. By the choice of $r, 2\left(\lambda_{2}+\beta_{1}\right) \mid \alpha_{r}, \mu_{r}$. Hence $\bar{h}^{\mu_{i}}=\bar{h}^{\mu_{r}} \in\left\langle\bar{h}^{2\left(\lambda_{2}+\beta_{1}\right)}\right\rangle$. From the last equation of (3.2), we have $\bar{h}^{\lambda_{2}+\beta_{1}+\mu_{i}}=\bar{h}^{\alpha_{i}}$ and $2\left(\lambda_{2}+\beta_{1}\right) \mid \alpha_{i}$. Hence we have $\bar{h}^{\lambda_{2}+\beta_{1}}=\bar{h}^{\alpha_{i}-\mu_{i}} \in\left\langle\bar{h}^{2\left(\lambda_{2}+\beta_{1}\right)}\right\rangle$, a contradiction. Thus $\bar{w} \bar{h}^{\lambda_{2}+\beta_{1}} \bar{b}_{i} \notin$ $\langle\bar{h}\rangle \bar{w} \bar{b}_{i}\langle\bar{h}\rangle$, and hence $\bar{c}_{1} \bar{d}_{1} \cdots \bar{c}_{i} \bar{d}_{i} \notin\langle\bar{h}\rangle \bar{a}_{1} \bar{b}_{1} \cdots \bar{a}_{i} \bar{b}_{i}\langle\bar{h}\rangle$. Therefore, $\bar{y} \notin\langle\bar{h}\rangle \bar{x}\langle\bar{h}\rangle$. Since $\bar{G}=A / M *\langle\bar{h}\rangle / N$ is residually finite, we can find $P \triangleleft_{f} G$ such that $y \notin P\langle h\rangle x\langle h\rangle$.

(c) $s \neq 0$ and $t \neq 0$. Clearly, $w^{-1} h^{i_{1}} w \notin\langle h\rangle$ for all $1 \leq i_{1}<s$ and $b_{i}^{-1} h^{j_{1}} b_{i} \notin$ $\langle h\rangle$ for all $1 \leq j_{1}<t$. Let $m=\operatorname{gcd}\{s, t\}$. Then $h^{\lambda_{2}+\beta_{1}} \notin\left\langle h^{s}\right\rangle\left\langle h^{t}\right\rangle=\left\langle h^{m}\right\rangle$. As before, we can find $M \triangleleft_{f} A$ and $N \triangleleft_{f} B$ such that in $\bar{G}=A / M *_{\langle\bar{h}\rangle} B / N$,

(1) $\|\bar{x}\|=\|x\|$ and $\bar{h}^{\lambda_{2}+\beta_{1}} \notin\left\langle\bar{h}^{m}\right\rangle=\left\langle\bar{h}^{s}\right\rangle\left\langle\bar{h}^{t}\right\rangle$,

(2) for each $1 \leq k \leq i, \bar{a}_{k}^{-1} \bar{h}^{i_{k}} \bar{a}_{k} \notin\langle\bar{h}\rangle$ for all $1 \leq i_{k}<n_{k}$, where $C_{\langle h\rangle}\left(a_{k}\right)=$ $\left\langle h^{n_{k}}\right\rangle$,

(3) for each $1 \leq k \leq i, \bar{b}_{k}^{-1} \bar{h}^{j_{k}} \bar{b}_{k} \notin\langle\bar{h}\rangle$ for all $1 \leq j_{k}<m_{k}$, where $C_{\langle h\rangle}\left(b_{k}\right)=$ $\left\langle h^{m_{k}}\right\rangle$, and

(4) if $\bar{h}^{i} \sim_{\bar{A}(\bar{B})} \bar{h}^{j}$, then $\bar{h}^{i}=\bar{h}^{j}$.

Then we have $\bar{w} \bar{h}^{\lambda_{2}+\beta_{1}} \bar{b}_{i} \notin\langle\bar{h}\rangle \bar{w} \bar{b}_{i}\langle\bar{h}\rangle$. For, if $\bar{w} \bar{h}^{\lambda_{2}+\beta_{1}} \bar{b}_{i} \in\langle\bar{h}\rangle \bar{w} \bar{b}_{i}\langle\bar{h}\rangle$, then there exist integers $\alpha_{i}, \mu_{i}$ such that the equations in (3.2) hold. As before, we have $\bar{h}^{\alpha_{1}}=\bar{h}^{\mu_{1}} \in C_{\langle\bar{h}\rangle}\left(\bar{a}_{1}\right)$. Similarly, $\bar{h}^{\mu_{1}}=\bar{h}^{\alpha_{2}} \in C_{\langle\overline{h\rangle}}\left(\bar{b}_{1}\right), \ldots, \bar{h}^{\alpha_{i}}=$ $\bar{h}^{\mu_{i}} \in C_{\langle\bar{h}\rangle}\left(\bar{a}_{i}\right)$. Hence $\bar{h}^{\alpha_{1}}=\bar{h}^{\mu_{1}}=\cdots=\bar{h}^{\mu_{i}} \in C_{\langle\bar{h}\rangle}\left(\bar{a}_{1} \bar{b}_{1} \cdots \bar{a}_{i}\right)=\left\langle\overline{h^{s}}\right\rangle$. From the last equation of (3.2), $\bar{h}^{\lambda_{2}+\beta_{1}+\mu_{i}}=\bar{h}^{\alpha_{i}} \in C_{\langle\bar{h}\rangle}\left(\bar{b}_{i}\right)=\left\langle\overline{h^{t}}\right\rangle$. Hence $\bar{h}^{\lambda_{2}+\beta_{1}}=\bar{h}^{\alpha_{i}-\mu_{i}} \in\left\langle\bar{h}^{s}\right\rangle\left\langle\bar{h}^{t}\right\rangle$, a contradiction. Thus $\bar{w}^{\lambda_{2}+\beta_{1}} \bar{b}_{i} \notin\langle\bar{h}\rangle \bar{w} \bar{b}_{i}\langle\bar{h}\rangle$, and hence $\bar{c}_{1} \bar{d}_{1} \cdots \bar{c}_{i} \bar{d}_{i} \notin\langle\bar{h}\rangle \bar{a}_{1} \bar{b}_{1} \cdots \bar{a}_{i} \bar{b}_{i}\langle\bar{h}\rangle$. Therefore, $\bar{y} \notin\langle\bar{h}\rangle \bar{x}\langle\bar{h}\rangle$. Since $\bar{G}=A / M *\langle\bar{h}\rangle=/ N$ is residually finite, we can find $P \triangleleft_{f} G$ such that $y \notin$ $P\langle h\rangle x\langle h\rangle$.

Lemma 3.6. Let $G$ be a group and $N$ be a finite normal subgroup of $G$. Let $S$ be a subset of $G$. Suppose that $G$ is $S$-separable. Then the group $G / N$ is $S / N$-separable, where $S / N=\{s N \mid s \in S\}$.

Proof. Let $x N \notin S / N$. Then $x n \notin S$ for every $n \in N$. Therefore, since $G$ is $S$-separable, there exists $M_{n} \triangleleft_{f} G$ such that $x n \notin M_{n} S$. Let $M=\cap_{n \in N} M_{n}$. Then $M \triangleleft_{f} G$ and $x n \notin M S$ for all $n \in N$. Thus $x N \notin(M N / N)(S / N)$ and $M N / N \triangleleft_{f} G / N$.

Definition 3.7. Let $G$ be a group and $\langle h\rangle \times D$ be a subgroup of $G$ with $|h|=\infty$. Then we say that $G$ satisfies $(C)$ for $\langle h\rangle \times D$ if there exists an integer $\delta>0$ such that for each $n>0$, there exists $M \triangleleft_{f} G$ depending on $n$ such that $D \subset M, M \cap\langle h\rangle=\left\langle h^{n \delta}\right\rangle$ and if $M h^{i} \sim_{G / M} M h^{j}$, then $M h^{i}=M h^{j}$. $\langle h\rangle$.

It is easy to see that if $G$ satisfies $(C)$ for $\langle h\rangle \times D$, then $G$ satisfies $\left(C^{\prime}\right)$ for 
Corollary 3.8. Let $G=A *_{H} B$ with $H=\langle h\rangle \times D$, where $|h|=\infty$ and $D \subset Z(A) \cap Z(B)$ is finite. Suppose $A, B$ are $\langle h\rangle$-self-conjugate, $\left\langle h^{n}\right\rangle$-double coset separable for each $n>0$. Let $A, B$ satisfy the condition $(C)$ for $\langle h\rangle \times D$. Then $G$ is $H$-double coset separable.

Proof. Let $y, x \in G$ and $y \notin H x H$. Let $\bar{G}=G / D$. Then $\bar{G}=\bar{A} * \bar{h}\rangle \bar{B}$, where $\bar{A}=A / D$ and $\bar{B}=B / D$. Since $D \subset H, \bar{y} \notin \bar{H} \bar{x} \bar{H}=\langle\bar{h}\rangle \bar{x}\langle\bar{h}\rangle$ in $\bar{G}$. By Lemma 3.6, $\bar{A}, \bar{B}$ are $\left\langle\bar{h}^{n}\right\rangle$-double coset separable for each $n>0$. To show that $\bar{A}$ is $\langle\bar{h}\rangle$-self-conjugate, suppose $\bar{h}^{i} \sim_{\bar{A}} \bar{h}^{j}$. Then $h^{i} \sim_{A} h^{j} d$ for some $d \in D$. Let $|d|=\alpha$. Then $h^{i \alpha} \sim_{A} h^{j \alpha} d^{\alpha}=h^{j \alpha}$. Since $A$ is $\langle h\rangle$-self-conjugate, we have $i \alpha=j \alpha$, that is, $i=j$. Hence $\bar{A}$ is $\langle\bar{h}\rangle$-self-conjugate. Similarly, $B$ is also $\langle\bar{h}\rangle$-self-conjugate. By Theorem 3.5, $\bar{G}=\bar{A} *\langle\bar{h}\rangle \bar{B}$ is $\langle\bar{h}\rangle \bar{x}\langle\bar{h}\rangle$-separable for all $\bar{x} \in \bar{G}$. There exists $\bar{P} \triangleleft_{f} \bar{G}$ such that $\bar{y} \notin \bar{P}\langle\bar{h}\rangle \bar{x}\langle\bar{h}\rangle=\overline{P H} \bar{x} \bar{H}$. Let $P$ be the preimage of $\bar{P}$. Then $P \triangleleft_{f} G$ and $y \notin P H x H$. Hence $G$ is $H x H$-separable for all $x \in G$, that is, $G$ is $H$-double coset separable.

\section{Criterion}

Theorem 4.1. Let $G=A *_{H} B$ with $H=\langle h\rangle \times D$, where $|h|=\infty$ and $D$ is a finite normal subgroup of both $A$ and $B$. Suppose there exists an integer $\delta_{1}>0$ such that for each $n>0$, there exists $M \triangleleft_{f} A$ such that $D \subset M$, $M \cap\langle h\rangle=\left\langle h^{n \delta_{1}}\right\rangle$. Similarly, suppose there exists an integer $\delta_{2}>0$ such that for each $n>0$, there exists $N \triangleleft_{f} B$ such that $D \subset N, N \cap\langle h\rangle=\left\langle h^{n \delta_{2}}\right\rangle$. If $A, B$ and $\tilde{G}=G / D$ are residually finite, then $G$ is residually finite.

Proof. Let $1 \neq x \in G$. We shall find $P \triangleleft_{f} G$ such that $x \notin P$.

(1) Let $x \notin D$. Then $1 \neq \tilde{x} \in \tilde{G}=G / D$. Since $\tilde{G}$ is residually finite, there exists $\tilde{P} \triangleleft_{f} \tilde{G}$ such that $\tilde{x} \notin \tilde{P}$. Let $P$ be the preimage of $\tilde{P}$. Then $P \triangleleft_{f} G$ and $x \notin P$.

(2) Let $x \in D$. Since $A, B$ are residually finite, there exist $M_{1} \triangleleft_{f} A$ and $N_{1} \triangleleft_{f} B$ such that $M_{1} \cap D=1=N_{1} \cap D$. Let $M_{1} \cap\langle h\rangle=\left\langle h^{s_{1}}\right\rangle$ and $N_{1} \cap$ $\langle h\rangle=\left\langle h^{s_{2}}\right\rangle$ for some $s_{1}, s_{2}$. By assumption, there exists $M_{2} \triangleleft_{f} A$ such that $M_{2} \cap\langle h\rangle=\left\langle h^{s_{1} s_{2} \delta_{1} \delta_{2}}\right\rangle$. Similarly, there exists $N_{2} \triangleleft_{f} B$ such that $N_{2} \cap\langle h\rangle=$ $\left\langle h^{s_{1} s_{2} \delta_{1} \delta_{2}}\right\rangle$. Let $M=M_{1} \cap M_{2}$ and $N=N_{1} \cap N_{2}$. Clearly, $M \triangleleft_{f} A, N \triangleleft_{f} B$ and $M \cap H=\left\langle h^{s_{1} s_{2} \delta_{1} \delta_{2}}\right\rangle=N \cap H$. Let $\bar{G}=A / M * \bar{H} B / N$, where $\bar{H}=\langle\bar{h}\rangle \times \bar{D}$. Then $\bar{G}$ is residually finite. Since $1 \neq \bar{x} \in \bar{D}$, there exists $\bar{P} \triangleleft_{f} \bar{G}$ such that $\bar{x} \notin \bar{P}$. Let $P$ be the preimage of $\bar{P}$. Then $P \triangleleft_{f} G$ and $x \notin P$.

In the above result, we note that the same conclusion can be drawn by assuming that $A, B$ are $H$-separable instead of the residual finiteness of $\tilde{G}=$ $G / D$.

Lemma 4.2. Let $A$ be a group and $D$ be a finite subgroup of $Z(A)$. Suppose $A$ is $\langle h\rangle$-self-conjugate. Let $a \in A$ and $a^{-1} h^{-i} a h^{i} \notin D$ for all $i \neq 0$. Then for 
each positive integer $m, h^{-i_{1}} a h^{j_{1}} d \notin\left\langle h^{m}\right\rangle a\left\langle h^{m}\right\rangle$ for all $0 \leq i_{1}, j_{1}<m$ and for all $d \in D$ except $i_{1}=j_{1}=0$ and $d=1$.

Proof. Let $0 \leq i_{1}, j_{1}<m$ and $d \in D$ such that $h^{-i_{1}} a h^{j_{1}} d=h^{k_{1} m} a h^{k_{2} m}$ for some $k_{1}, k_{2}$. Then $a^{-1} h^{i_{1}+k_{1} m} a=h^{j_{1}-k_{2} m} d$. Let $|d|=\alpha$. Then

$$
a^{-1} h^{\left(i_{1}+k_{1} m\right) \alpha} a=h^{\left(j_{1}-k_{2} m\right) \alpha} d^{\alpha}=h^{\left(j_{1}-k_{2} m\right) \alpha} .
$$

Since $A$ is $\langle h\rangle$-self-conjugate, $\left(i_{1}+k_{1} m\right) \alpha=\left(j_{1}-k_{2} m\right) \alpha$, and hence $i_{1}+$ $k_{1} m=j_{1}-k_{2} m$. Thus $a^{-1} h^{i_{1}+k_{1} m} a=h^{j_{1}-k_{2} m} d=h^{i_{1}+k_{1} m} d$. By assumption, $i_{1}+k_{1} m=0$. This implies that $i_{1}=j_{1}=0$ and $d=1$.

Lemma 4.3. Let $G=A *_{H} B$ with $H=\langle h\rangle \times D$, where $|h|=\infty$ and $D \subset$ $Z(A) \cap Z(B)$ is finite. Let $A, B$ be $\langle h\rangle$-self-conjugate and $\left\langle h^{n}\right\rangle$-double coset separable for each $n>0$ and let $A, B$ satisfy $(C)$ above. Let $x=a_{1} b_{1} \cdots a_{n} b_{n}$, where $a_{i} \in A \backslash H, b_{i} \in B \backslash H$. If $x \not_{H} x u$, where $u \in D$, then there exist $M \triangleleft_{f} A$ and $N \triangleleft_{f} B$ such that $M \cap H=N \cap H$ and in $\bar{G}=A / M * \bar{H} B / N,\|\bar{x}\|=\|x\|$ and $\bar{x} \chi_{\bar{H}} \overline{x u}$.

Proof. Case 1. Suppose there is a positive integer $s$ such that $x^{-1} h^{-s} x h^{s} \in D$. We can assume that $s$ is the least among such integers and $x^{-1} h^{-s} x h^{s}=c \in D$. Then $h^{-k s} x h^{k s}=x c^{k}$ for integer $k$. Since $x \chi_{H} x u$, we have $u \notin\langle c\rangle$. By the minimality of $s$, we have $x^{-1} h^{-i_{1}} x h^{i_{1}} \notin D$ for all $1 \leq i_{1} \leq s-1$. Note that $G$ is residually finite (Theorem 4.1). Hence there exists $P \triangleleft_{f} G$ such that $x^{-1} h^{-i_{1}} x h^{i_{1}} \notin P D$ for all $1 \leq i_{1} \leq s-1$ and $u \notin P\langle c\rangle$ ( $D$ is finite, so $\langle c\rangle$ is finite). Let $M=P \cap A$ and $N=P \cap B$. Then $M \cap H=N \cap H$. In $\bar{G}=A / M *_{\bar{H}} B / N$, we shall prove that $\bar{x} \chi_{\bar{H}} \overline{x u}$.

Suppose $\bar{x} \sim_{\bar{H}} \overline{x u}$. Then $\overline{x u}=\bar{h}^{-i} \bar{x} \bar{h}^{i}$ for some $i$. Let $i=k s+i_{1}$, where $0 \leq i_{1}<s$. Then $\overline{x u}=\bar{h}^{-i} \bar{x}^{i}=\bar{h}^{-i_{1}} \bar{h}^{-k s} \bar{x}^{k s} \bar{h}^{i_{1}}=\bar{h}^{-i_{1}} \overline{x c}^{k} \bar{h}^{i_{1}}$. Hence $\bar{x}^{-1} \bar{h}^{-i_{1}} \bar{x}^{i_{1}}=\overline{u c}^{-k} \in \bar{D}$. By the choice of $P, i_{1}=0$. Then $\bar{u}=\bar{c}^{k} \in\langle\bar{c}\rangle$, a contradiction. Therefore, we have $\bar{x} \chi_{\bar{H}} \overline{x u}$.

Case 2. Suppose there is no positive integer $s$ such that $x^{-1} h^{-s} x h^{s} \in D$.

In this case, there exists either $a_{r}$ such that $a_{r}^{-1} h^{-i} a_{r} h^{i} \notin D$ for all $i \neq 0$ or $b_{r}$ such that $b_{r}^{-1} h^{-i} b_{r} h^{i} \notin D$ for all $i \neq 0$. Note that $x \sim_{H} x u$ if and only if $x^{*} \sim_{H} x^{*} u$ for any cyclic permutation $x^{*}$ of $x$. Hence, without loss of generality, we may assume $a_{1}^{-1} h^{-i} a_{1} h^{i} \notin D$ for all $i \neq 0$.

Suppose $a_{\ell}^{-1} h^{-s} a_{\ell} h^{s} \in D$ for some integer $s \neq 0$, say $h^{-s} a_{\ell} h^{s}=a_{\ell} w_{\ell}$ for some $w_{\ell} \in D$. Since $D$ is finite, $h^{-s k} a_{\ell} h^{s k}=a_{\ell} w_{\ell}^{k}=a_{\ell}$ for some $k$. Thus if $a_{\ell}^{-1} h^{-s} a_{\ell} h^{s} \in D$ for some $s \neq 0$, then there exists smallest positive integer $s_{\ell}$ such that $h^{-s_{\ell}} a_{\ell} h^{s_{\ell}}=a_{\ell}$. Similarly, if $b_{\ell}^{-1} h^{-s} b_{\ell} h^{s} \in D$ for some integer $s \neq 0$, then there exists smallest positive integer $t_{\ell}$ such that $h^{-t_{\ell}} b_{\ell} h^{t_{\ell}}=b_{\ell}$. Let

$$
\begin{aligned}
I & =\left\{\ell \mid a_{\ell}=h^{-s_{\ell}} a_{\ell} h^{s_{\ell}} \text { for some minimal } s_{\ell}>0\right\} \text { and } \\
J & =\left\{\ell \mid b_{\ell}=h^{-t_{\ell}} b_{\ell} h^{t_{\ell}} \text { for some minimal } t_{\ell}>0\right\} .
\end{aligned}
$$


Of course, $I, J$ can be empty. But $1 \notin I$ by the assumption in the beginning of Case 2. Let $s=\prod_{i \in I} s_{i}$ and $t=\prod_{i \in J} t_{i}$, where $s=1$ if $I=\emptyset$ and $t=1$ if $J=\emptyset$. Let $m=s t$.

Since $A$ is $\langle h\rangle$-separable and $D$ is finite, $A$ is $H$-separable. Similarly, $B$ is $H$-separable. There exists $M_{1} \triangleleft_{f} A$ such that $M_{1} \cap D=1$ and $a_{\lambda} \notin M_{1} H$ for all $1 \leq \lambda \leq n$. Thus $u \notin M_{1}$. Similarly, there exists $N_{1} \triangleleft_{f} B$ such that $N_{1} \cap D=1$ and $b_{\lambda} \notin N_{1} H$ for all $1 \leq \lambda \leq n$.

For those $a_{r}$ where $r \notin I, a_{r}^{-1} h^{-i} a_{r} h^{i} \notin D$ for all $i \neq 0$. Thus by Lemma 4.2, $h^{-i_{1}} a_{r} h^{j_{1}} d \notin\left\langle h^{m}\right\rangle a_{r}\left\langle h^{m}\right\rangle$ for all $0 \leq i_{1}, j_{1}<m$ and for all $d \in D$ except $i_{1}=j_{1}=0$ and $d=1$. Since $A$ is $\left\langle h^{m}\right\rangle$-double coset separable, there exists $M_{2} \triangleleft_{f} A$ such that for all possible $r \notin I, h^{-i_{1}} a_{r} h^{j_{1}} d \notin M_{2}\left\langle h^{m}\right\rangle a_{r}\left\langle h^{m}\right\rangle$ for all $0 \leq i_{1}, j_{1}<m$ and for all $d \in D$ except $i_{1}=j_{1}=0$ and $d=1$. Note that $D \cap M_{2}=1$. Then in $\tilde{A}=A / M_{2}$, if $\tilde{a}_{r}=\tilde{h}^{i} \tilde{a}_{r} \tilde{h}^{j} \tilde{d}$ for $d \in D$, then $m \mid i, j$ and $d=1$. For those $b_{r}$ where $r \notin J$, we have $h^{-i} b_{r}^{-1} h^{i} b_{r} \notin D$ for all $i \neq 0$. Then, as before, there exists $N_{2} \triangleleft_{f} B$ such that for all possible $r \notin J$, if $\tilde{b}_{r}=\tilde{h}^{i} \tilde{b}_{r} \tilde{h}^{j} \tilde{d}$ where $\tilde{B}=B / N_{2}$ and $d \in D$, then $m \mid i, j$ and $d=1$.

Let $M_{1} \cap M_{2} \cap\langle h\rangle=\left\langle h^{s_{1}}\right\rangle$ and $N_{1} \cap N_{2} \cap\langle h\rangle=\left\langle h^{s_{2}}\right\rangle$. By $(C)$, there exist $M_{3} \triangleleft_{f} A$ and $N_{3} \triangleleft_{f} B$ such that $M_{3} \cap H=\left\langle h^{s_{1} s_{2}} \delta_{1} \delta_{2}\right\rangle \times D=N_{3} \cap H$. Let $M=M_{1} \cap M_{2} \cap M_{3}$ and $N=N_{1} \cap N_{2} \cap N_{3}$. In $\bar{G}=A / M * \bar{H} B / N$, where $\bar{H}=\langle\bar{h}\rangle \times \bar{D}$, we shall prove that $\bar{x} \chi_{\bar{H}} \overline{x u}$.

Suppose $\bar{x} \sim_{\bar{H}} \overline{x u}$. There exist $\alpha_{i}, \mu_{i}$ and $c_{i}, d_{i} \in D$ such that

$$
\begin{gathered}
\bar{a}_{1}=\bar{h}^{-\alpha_{1}} \bar{d}_{1}^{-1} \bar{a}_{1} \bar{h}^{\mu_{1}} \bar{c}_{1} \\
\bar{b}_{1}=\bar{h}^{-\mu_{1}} \bar{c}_{1}^{-1} \bar{b}_{1} \bar{h}^{\alpha_{2}} \bar{d}_{2} \\
\bar{a}_{2}=\bar{h}^{-\alpha_{2}} \bar{d}_{2}^{-1} \bar{a}_{2} \bar{h}^{\mu_{2}} \bar{c}_{2} \\
\vdots \\
\bar{b}_{n} \bar{u}=\bar{h}^{-\mu_{n}} \bar{c}_{n}^{-1} \bar{b}_{n} \bar{h}^{\alpha_{1}} \bar{d}_{1} .
\end{gathered}
$$

From the first equation in (4.3), we have $\bar{a}_{1}=\bar{h}^{-\alpha_{1}} \bar{a}_{1} \bar{h}^{\mu_{1}} \bar{d}_{1}^{-1} \bar{c}_{1}$. Since $1 \notin I$, by the choice of $M_{2}$ we have $m \mid-\alpha_{1}, \mu_{1}$ and $d_{1}^{-1} c_{1}=1$. If $1 \notin J$ again, we can easily see that $c_{1}^{-1} d_{2}=1$. So suppose $1 \in J$, that is, there exists a minimal positive integer $t_{1}$ such that $b_{1}=h^{-t_{1}} b_{1} h^{t_{1}}$. Thus $h^{-t_{1} k} b_{1} h^{t_{1} k}=b_{1}$ for all integer $k$. From the second equation of (4.3), we have $\bar{b}_{1}=\bar{h}^{-\mu_{1}} \bar{b}_{1} \bar{h}^{\mu_{1}} \bar{h}^{-\mu_{1}+\alpha_{2}} \bar{a}_{1}^{-1} \bar{d}_{2}$. Since $m \mid \mu_{1}$, let $\mu_{1}=m c=\left(\prod_{i \in I} s_{i}\right)\left(\prod_{i \in J} t_{i}\right) c$. Then $\bar{b}_{1}=\bar{b}_{1} \bar{h}^{-\mu_{1}+\alpha_{2}} \bar{a}_{1}^{-1} \bar{d}_{2}$. Hence $\bar{h}^{-\mu_{1}+\alpha_{2}} \bar{a}_{1}^{-1} \bar{d}_{2}=1$. Since $\langle\bar{h}\rangle \cap \bar{D}=1$, we have $\bar{h}^{\mu_{1}}=\bar{h}^{\alpha_{2}}$ and $\bar{a}_{1}^{-1} \bar{d}_{2}=1$.

By a similar way, we can see that all $\bar{d}_{i}^{-1} \bar{c}_{i}=1$ and $\bar{c}_{i}^{-1} \bar{d}_{i+1}=1$. From the last equation in (4.3), we have $\bar{b}_{n}=\bar{h}^{-\mu_{n}} \bar{b}_{n} \bar{h}^{\alpha_{1}} \bar{c}_{n}^{-1} \bar{d}_{1} \bar{u}^{-1}$. As before, we can prove that $\bar{c}_{n}^{-1} \bar{d}_{1} \bar{u}^{-1}=1$. Then

$$
\bar{u}^{-1}=\bar{d}_{1}^{-1} \bar{c}_{1} \cdot \bar{c}_{1}^{-1} \bar{d}_{2} \cdots \bar{d}_{i}^{-1} \bar{c}_{i} \cdot \bar{c}_{i}^{-1} \bar{d}_{i+1} \cdots \bar{c}_{n}^{-1} \bar{d}_{1} \bar{u}^{-1}=1,
$$


a contradiction by the choice of $M_{1}$. This proves that $\bar{x} \chi_{\bar{H}} \overline{x u}$.

Definition 4.4. Let $G$ be a group and let $H$ be a subgroup of $G$. We say $G$ is $H$-conjugacy separable if for each $x \in G$ such that $\{x\}^{G} \cap H=\emptyset$, there exists $N \triangleleft_{f} G$ such that in $\bar{G}=G / N,\{\bar{x}\}^{\bar{G}} \cap \bar{H}=\emptyset$.

Dyer [4] noted the importance of the above notion in the study of conjugacy separability of generalized free products, that is, if $G$ has a subgroup $H$ and is not $H$-conjugacy separable, then $G *_{H} G$ is not conjugacy separable.

Now we are ready to prove our main result.

Theorem 4.5. Let $G=A *_{H} B$ with $H=\langle h\rangle \times D$, where $|h|=\infty$ and $D \subset Z(A) \cap Z(B)$ is finite, such that $\tilde{G}=G / D$ is conjugacy separable. Let $A, B$ be conjugacy separable, $\langle h\rangle$-self-conjugate, and $\left\langle h^{n}\right\rangle$-double coset separable for each $n>0$ and $H$-conjugacy separable. If $A, B$ satisfy $(C)$, then $G$ is conjugacy separable.

Proof. Let $x, y \in G$ be elements of minimal lengths in their conjugate classes and $x \nsim_{G} y$. Since $\tilde{G}=G / D=\tilde{A} *_{\langle\tilde{h}\rangle} \tilde{B}$ is conjugacy separable, if $\tilde{x} \varkappa_{\tilde{G}} \tilde{y}$, then we can find $\tilde{P} \triangleleft_{f} \tilde{G}$ such that $\tilde{P} \tilde{x} \chi_{\tilde{G} / \tilde{P}} \tilde{P} \tilde{y}$. Let $P$ be the preimage of $\tilde{P}$. Then $P \triangleleft_{f} G$ and $P x \varkappa_{G / P} P y$. Hence we assume that $\tilde{x} \sim_{\tilde{G}} \tilde{y}$. Then $y \sim_{G} x u$ for some $u \in D$. Hence we can take $y=x u$ and $x \varkappa_{G} x u$. We shall find $M \triangleleft_{f} A$ and $N \triangleleft_{f} B$ such that in $\bar{G}=A / M * \bar{H} B / N, \bar{x} \chi_{\bar{G}} \overline{x u}$. Since $\bar{G}$ is conjugacy separable, there exists $\bar{P} \triangleleft_{f} \bar{G}$ such that $\bar{P} \bar{x} \chi_{\bar{G}} / \bar{P} \bar{P} \overline{x u}$. Let $P$ be the preimage of $\bar{P}$. Then $P \triangleleft_{f} G$ and $P x \varkappa_{G / P} P y$. Hence $G$ is conjugacy separable.

Case 1. $x \in H$. Without loss of generality, we assume $x=h^{\alpha}$. Then $h^{\alpha} \chi_{A} h^{\alpha} u$ and $h^{\alpha} \varkappa_{B} h^{\alpha} u$. Since $A$ is conjugacy separable and $D$ is finite, there exists $M_{1} \triangleleft_{f} A$ such that $M_{1} \cap D=1$ and $M_{1} h^{\alpha} \nsim M_{1} h^{\alpha} d$ for all $d \in D$ such that $h^{\alpha} \chi_{A} h^{\alpha} d$. Similarly, there exists $N_{1} \triangleleft_{f} B$ such that $N_{1} \cap D=1$ and $N_{1} h^{\alpha} \nsim N_{1} h^{\alpha} d$ for all $d \in D$ such that $h^{\alpha} \chi_{B} h^{\alpha} d$. Let $M_{1} \cap\langle h\rangle=\left\langle h^{s_{1}}\right\rangle$ and $N_{1} \cap\langle h\rangle=\left\langle h^{s_{2}}\right\rangle$ for some integers $s_{1}, s_{2}$. By $(C)$, for $n=s_{1} s_{2} \delta_{2}$, there exists $M_{2} \triangleleft_{f} A$ such that $D \subset M_{2}, M_{2} \cap\langle h\rangle=\left\langle h^{s_{1} s_{2} \delta_{1} \delta_{2}}\right\rangle$ and if $M_{2} h^{i} \sim_{A / M_{2}} M_{2} h^{j}$, then $M_{2} h^{i}=M_{2} h^{j}$. Similarly, for $n=s_{1} s_{2} \delta_{1}$, there exists $N_{2} \triangleleft_{f} B$ such that $D \subset N_{2}, N_{2} \cap\langle h\rangle=\left\langle h^{s_{1} s_{2} \delta_{1} \delta_{2}}\right\rangle$ and if $N_{2} h^{i} \sim_{B / N_{2}} N_{2} h^{j}$, then $N_{2} h^{i}=N_{2} h^{j}$. Let $M=M_{1} \cap M_{2}$ and $N=N_{1} \cap N_{2}$. Clearly, $M \cap H=\left\langle h^{s_{1} s_{2} \delta_{1} \delta_{2}}\right\rangle=N \cap H$. In $\bar{G}=A / M * \bar{H} B / N$, where $\bar{H}=\langle\bar{h}\rangle \times \bar{D}$, we show that $\bar{h}^{\alpha} \chi_{\bar{G}} \bar{h}^{\alpha} \bar{u}$.

Suppose $\bar{h}^{\alpha} \sim_{\bar{G}} \bar{h}^{\alpha} \bar{u}$. Then by Theorem 2.2, there exist integers $c_{i}$ and $d_{i} \in D$ such that

$$
\bar{h}^{\alpha} \sim_{\bar{A}} \bar{h}^{c_{1}} \bar{d}_{1} \sim_{\bar{B}} \bar{h}^{c_{2}} \bar{d}_{2} \sim_{\bar{A}} \cdots \sim_{\bar{B}(\bar{A})} \bar{h}^{c_{n}} \bar{d}_{n} \sim_{\bar{A}(\bar{B})} \bar{h}^{\alpha} \bar{u} .
$$

From the first conjugate relation $\bar{h}^{\alpha} \sim \bar{h}^{c_{1}} \bar{d}_{1}$, we have $M_{2} h^{\alpha} \sim M_{2} h^{c_{1}}$. Hence, by the choice of $M_{2}$, we have $M_{2} h^{\alpha}=M_{2} h^{c_{1}}$. Thus $h^{\alpha-c_{1}} \in M_{2} \cap\langle h\rangle=M \cap\langle h\rangle$, whence $\bar{h}^{\alpha}=\bar{h}^{c_{1}}$. It follows that $\bar{h}^{\alpha} \sim \bar{A} \bar{h}^{\alpha} \bar{d}_{1}$. Hence $M_{1} h^{\alpha} \sim M_{1} h^{\alpha} d_{1}$. By the choice of $M_{1}$, we have $h^{\alpha} \sim_{A} h^{\alpha} d_{1}$. From the second conjugate relation 
of (4.4), we have $\bar{h}^{\alpha}=\bar{h}^{c_{1}} \sim_{\bar{B}} \bar{h}^{c_{2}} \bar{d}_{2} \bar{d}_{1}^{-1}$. Then, as before, $\bar{h}^{\alpha}=\bar{h}^{c_{2}}$ and $h^{\alpha} \sim_{B} h^{\alpha} d_{2} d_{1}^{-1}$. Hence $h^{\alpha} d_{1} \sim_{B} h^{\alpha} d_{2}$. Similarly, from (4.4), we have $\bar{h}^{\alpha}=\bar{h}^{c_{n}}$ and $h^{\alpha} d_{n} \sim_{A(B)} h^{\alpha} u$. Thus we have

$$
h^{\alpha} \sim_{A} h^{\alpha} d_{1} \sim_{B} \cdots \sim_{B(A)} h^{\alpha} d_{n} \sim_{A(B)} h^{\alpha} u .
$$

It follows that $h^{\alpha} \sim_{G} h^{\alpha} u$, a contradiction. Therefore, $\bar{h}^{\alpha} \chi_{\bar{G}} \bar{h}^{\alpha} \bar{u}$.

Case 2. $x \in A \backslash H$ (or $x \in B \backslash H$ ). Let $x \in A$ and $x$ have the minimal length 1 in its conjugacy class in $G$. Let $x \not_{G} x u$, where $u \in D$. Since $x$ has the minimal length 1 in its conjugacy class, $x \chi_{A} h^{i} d$ for all $i$ and $d \in D$. Hence, by $H$ conjugacy separability, there exists $M_{1} \triangleleft_{f} A$ such that $M_{1} x \nsim_{A / M_{1}} M_{1} h^{i} d$ for all $i$ and all $d \in D$. Since $A$ is conjugacy separable, there exists $M_{2} \triangleleft_{f} A$ such that $M_{2} \cap D=1$ and $M_{2} x \nsim_{A / M_{2}} M_{2} x d$ for all $d \in D$ such that $x \nsim_{A} x d$. Similarly, since $B$ is conjugacy separable, there exists $N_{1} \triangleleft_{f} B$ such that $N_{2} \cap D=1$. Let $M_{1} \cap M_{2} \cap\langle h\rangle=\left\langle h^{s_{1}}\right\rangle$ and $N_{1} \cap\langle h\rangle=\left\langle h^{s_{2}}\right\rangle$ for some $s_{1}, s_{2}$. By $(C)$, there exist $M_{3} \triangleleft_{f} A$ and $N_{2} \triangleleft_{f} B$ such that $D \subset M_{3}, D \subset N_{2}$ and $M_{3} \cap\langle h\rangle=\left\langle h^{s_{1} s_{2} \delta_{1} \delta_{2}}\right\rangle=$ $N_{2} \cap\langle h\rangle$.

Let $M=M_{1} \cap M_{2} \cap M_{3}$ and $N=N_{1} \cap N_{2}$. Then $M \cap H=\left\langle h^{s_{1} s_{2} \delta_{1} \delta_{2}}\right\rangle=N \cap H$. In $\bar{G}=A / M * \bar{H} B / N$, where $\bar{H}=\langle\bar{h}\rangle \times \bar{D}$, we shall prove that $\bar{x} \chi_{\bar{G}} \overline{x u}$.

Suppose $\bar{x} \sim_{\bar{G}} \overline{x u}$. By the choice of $M_{1}, \bar{x}$ is not conjugate to any element in $\bar{H}$. Hence $\bar{x}$ has the minimal length 1 in its conjugacy class in $\bar{G}$. It follows from Theorem 2.2 that $\bar{x} \sim_{\bar{A}} \overline{x u}$. Then by choice of $M_{2}, x \sim_{A} x u$, a contradiction. Therefore, $\bar{x} \chi_{\bar{G}} \overline{x u}$.

Case 3. $x=a_{1} b_{1} \cdots a_{n} b_{n}$ (or $x=b_{1} a_{1} \cdots b_{n} a_{n}$ ), where $a_{i} \in A \backslash H$ and $b_{i} \in B \backslash H$. Let $x \chi_{G} x u$, where $u \in D$. Let $x_{i}=a_{i} b_{i} \cdots a_{n} b_{n} a_{1} b_{1} \cdots a_{i-1} b_{i-1}$ for $1 \leq i \leq n$. Clearly, $x=x_{1}$. By Theorem 2.2, $x_{i} \not_{H} x u$ for all $1 \leq i \leq$ $n$. We shall find $M_{i} \triangleleft_{f} A$ and $N_{i} \triangleleft_{f} B$ such that $M_{i} \cap H=N_{i} \cap H$ and in $\bar{G}=A / M_{i} *_{\bar{H}} B / N_{i},\left\|\bar{x}_{i}\right\|=2 n=\|x\|$ and $\bar{x}_{i} \chi_{\bar{H}} \overline{x u}$ for each $1 \leq i \leq n$. The case of $i=1$ is done by Lemma 4.3. Thus let $M_{1} \triangleleft_{f} A$ and $N_{1} \triangleleft_{f} B$ such that $M_{1} \cap H=N_{1} \cap H$ and in $\bar{G}=A / M_{1} * \bar{H} B / N_{1},\left\|\bar{x}_{1}\right\|=2 n=\|x\|$ and $\bar{x}_{1} \chi_{\bar{H}} \overline{x u}$. We shall consider the case when $i>1$.

Suppose $x_{i} \notin H x u H$. Then $x_{i} \notin H x H$. By Corollary 3.8, there exists $Q \triangleleft_{f} G$ such that $x_{i} \notin Q H x H, a_{i} \notin Q H$ and $b_{i} \notin Q H$ for all $1 \leq i \leq n$. Let $M_{i}=Q \cap A$ and $N_{i}=Q \cap B$. Then in $\bar{G}=A / M_{i} * \bar{H} B / N_{i},\left\|\bar{x}_{i}\right\|=2 n=\|x\|$ and $\bar{x}_{i} \notin \bar{H} \bar{x} \bar{H}=\bar{H} \overline{x u} \bar{H}$. Hence $\bar{x}_{i} \chi_{\bar{H}} \overline{x u}$ as required.

Suppose $x_{i} \in H x u H$, say $x_{i}=h_{1} x h_{2}$ for $h_{1}, h_{2} \in H$. Clearly, $x_{i} \sim_{H} x h_{2} h_{1}$. Hence $x h_{2} h_{1} \chi_{H} x u$. Let $h_{2} h_{1}=h^{s} u_{1}$, where $u_{1} \in D$.

(a) $s=0$. Then we have $x u_{1} \chi_{H} x u$, or equivalently, $x \nsim_{H} x u u_{1}^{-1}$, where $u u_{1}^{-1} \in D$. By Lemma 4.3, there exist $M_{i} \triangleleft_{f} A$ and $N_{i} \triangleleft_{f} B$ such that $M_{i} \cap H=$ $N_{i} \cap H$ and in $\bar{G}=A / M_{i} *_{H} B / N_{i},\|\bar{x}\|=2 n=\|x\|$ and $\bar{x} \chi_{\bar{H}} \overline{x u u}_{1}^{-1}$. Then $\bar{x}_{i} \sim_{\bar{H}} \overline{x u}_{1} \chi_{\bar{H}} \overline{x u}$, as required.

(b) $s \neq 0$. By Theorem 4.1 and Corollary 3.8, there exists $Q \triangleleft_{f} G$ such that $h^{s} \notin Q, a_{i} \notin Q H$ and $b_{i} \notin Q H$ for all $1 \leq i \leq n$. Let $P \cap\langle h\rangle=\left\langle h^{s_{1}}\right\rangle$. By $(C)$, there exists $M \triangleleft_{f} A$ such that $D \subset M, M \cap\langle h\rangle=\left\langle h^{s s_{1} \delta_{1} \delta_{2}}\right\rangle$ and if $M h^{i} \sim_{A / M}$ 
$M h^{j}$, then $M h^{i}=M h^{j}$. Similarly, there exists $N \triangleleft_{f} B$ such that $D \subset N$, $N \cap\langle h\rangle=\left\langle h^{s s_{1} \delta_{1} \delta_{2}}\right\rangle$ and if $N h^{i} \sim_{B / N} N h^{j}$, then $N h^{i}=N h^{j}$. Let $M_{i}=Q \cap M$, $N_{i}=Q \cap N$ and $\bar{G}=A / M_{i} * \bar{H} B / N_{i}$. Clearly, $M_{i} \cap\langle h\rangle=\left\langle h^{s s_{1} \delta_{1} \delta_{2}}\right\rangle=N_{i} \cap\langle h\rangle$ and $\left\|\bar{x}_{i}\right\|=2 n=\|x\|$. We shall show that $\bar{x}_{i} \chi_{\bar{H}} \overline{x u}$. Suppose $\bar{x}_{i} \sim_{\bar{H}} \overline{x u}$. Then $\bar{x} \bar{h}^{s} \bar{u}_{1} \sim \bar{H} \overline{x u}$. Hence there exist $\alpha_{i}, \mu_{i}$ and $d_{i}, c_{i} \in D$ such that

$$
\begin{gathered}
\bar{a}_{1}=\bar{h}^{-\alpha_{1}} \bar{d}_{1}^{-1} \bar{a}_{1} \bar{h}^{\mu_{1}} \bar{c}_{1} \\
\bar{b}_{1}=\bar{h}^{-\mu_{1}} \bar{c}_{1}^{-1} \bar{b}_{1} \bar{h}^{\alpha_{2}} \bar{d}_{2} \\
\bar{a}_{2}=\bar{h}^{-\alpha_{2}} \bar{d}_{2}^{-1} \bar{a}_{2} \bar{h}^{\mu_{2}} \bar{c}_{2} \\
\vdots \\
\bar{b}_{n} \bar{h}^{s} \bar{u}_{1}=\bar{h}^{-\mu_{n}} \bar{c}_{n}^{-1} \bar{b}_{n} \bar{u} \bar{h}^{\alpha_{1}} \bar{d}_{1} .
\end{gathered}
$$

From the first equation in (4.5), we have $M a_{1}=M h^{-\alpha_{1}} a_{1} h^{\mu_{1}}$, that is, $M h^{\alpha_{1}} \sim$ $M h^{\mu_{1}}$. By the choice of $M$, we have $M h^{\alpha_{1}}=M h^{\mu_{1}}$. Thus $h^{\mu-\alpha_{1}} \in M \cap\langle h\rangle \subset$ $P$. Therefore, $\bar{h}^{\alpha_{1}}=\bar{h}^{\mu_{1}}$. Similarly, from the second equation of (4.3), we have $\bar{h}^{\mu_{1}}=\bar{h}^{\alpha_{2}}$. Moreover, we have $\bar{h}^{\alpha_{2}}=\bar{h}^{\mu_{2}}, \bar{h}^{\mu_{2}}=\bar{h}^{\alpha_{3}}, \ldots, \bar{h}^{\alpha_{n}}=\bar{h}^{\mu_{n}}$, and $\bar{h}^{\mu_{n}}=\bar{h}^{\alpha_{1}-s}$. Therefore, $\bar{h}^{\alpha_{1}}=\bar{h}^{\alpha_{1}-s}$, which implies $\bar{h}^{s}=1$, a contradiction. Hence $\bar{x}_{i} \chi_{\bar{H}} \overline{x u}$ as required.

\section{Generalized free products of nilpotent groups}

In this section we apply the main result to finitely generated nilpotent groups.

Lemma 5.1. Let $A$ be finitely generated nilpotent and $h \in A$ with $|h|=\infty$. Then there exists a positive integer $\delta$ such that for each $n>0$, there exists $M \triangleleft_{f} A$ such that $M \cap\langle h\rangle=\left\langle h^{n \delta}\right\rangle$ and if $M h^{i} \sim_{A / M} M h^{j}$, then $M h^{i}=M h^{j}$.

Proof. Since $A$ is finitely generated nilpotent, there exists an integer $i \geq 0$ such that $Z_{i}(A) \cap\langle h\rangle=1$ and $Z_{i+1}(A) \cap\langle h\rangle=\left\langle h^{\delta}\right\rangle$ for some $\delta>0$. In $\hat{A}=A / Z_{i}(A)$, $|\hat{h}|=\infty$ and $\hat{h}^{\delta} \in Z(\hat{A})$. For a given integer $n>0$, consider $\bar{A}=\hat{A} /\left\langle\hat{h}^{n \delta}\right\rangle$. Clearly, $|\bar{h}|=n \delta$. If $\bar{h}^{i} \sim_{\bar{A}} \bar{h}^{j}$, then $\hat{h}^{j}=\hat{g}^{-1} \hat{h}^{i} \hat{g} \hat{h}^{k n \delta}=\hat{g}^{-1} \hat{h}^{i+k n \delta} \hat{g}$ for some $g \in A$ and for some integer $k$. Since $\hat{A}$ is finitely generated nilpotent and $|\hat{h}|=\infty$, we have $j=i+k n \delta$. Thus $\bar{h}^{i}=\bar{h}^{j}$. This shows that if $\bar{h}^{i} \sim_{\bar{A}} \bar{h}^{j}$, then $\bar{h}^{i}=\bar{h}^{j}$. Since $\bar{A}$ is finitely generated nilpotent, $\bar{A}$ is conjugacy separable. There exists $\bar{M} \triangleleft_{f} \bar{A}$ such that $\overline{M h}^{i} \chi_{\bar{A} / \bar{M}} \overline{M h}^{j}$ for all $\bar{h}^{i} \chi_{\bar{A}} \bar{h}^{j}$ (there are only finitely many). Considering $j=0$, we have $1 \neq \bar{h}^{i} \notin \bar{M}$. Let $M$ be the preimage of $\bar{M}$. Then $M \triangleleft_{f} A, M \cap\langle h\rangle=\left\langle h^{n \delta}\right\rangle$, and if $M h^{i} \sim_{A / M} M h^{j}$, then $M h^{i}=M h^{j}$. 
The above result proves that finitely nilpotent groups satisfy $\left(C^{\prime}\right)$ in Theorem 3.5. The next shows that those nilpotent groups satisfy $(C)$ in Definition 3.7 .

Lemma 5.2. Let $A$ be finitely generated nilpotent and $D \subset Z(A)$. Let $h \in A$ with $|h|=\infty$ and $D \cap\langle h\rangle=1$. Then there exists a positive integer $\delta$ such that for each $n>0$, there exists $M \triangleleft_{f} A$ such that $D \subset M, M \cap\langle h\rangle=\left\langle h^{n \delta}\right\rangle$, and if $M h^{i} \sim_{A / M} M h^{j}$, then $M h^{i}=M h^{j}$.

Proof. Since $\bar{A}=A / D$ is finitely generated nilpotent and $|\bar{h}|=\infty$, by Lemma 5.1 , there exists a positive integer $\delta$ such that for each $n>0$, there exists $\bar{M} \triangleleft_{f} \bar{A}$ such that $\bar{M} \cap\langle\bar{h}\rangle=\left\langle\bar{h}^{n \delta}\right\rangle$ and if $\overline{M h}^{i} \sim_{\bar{A}} / \bar{M} \overline{M h}^{j}$, then $\overline{M h}^{i}=\overline{M h}^{j}$. Let $M$ be the preimage of $\bar{M}$. Then $M \triangleleft_{f} A, D \subset M, M \cap\langle h\rangle=\left\langle h^{n \delta}\right\rangle$, and if $M h^{i} \sim_{A / M} M h^{j}$, then $M h^{i}=M h^{j}$.

Theorem 5.3. Let $G=A *_{H} B$ with $H=\langle h\rangle \times D$, where $|h|=\infty$ and $D \subset Z(A) \cap Z(B)$ is finite. If $A, B$ are finitely generated nilpotent, then $G$ is conjugacy separable.

Proof. Finitely generated nilpotent groups are conjugacy separable $[6,15]$ and cyclic conjugacy separable [4]. Hence $A, B$ are conjugacy separable and $\tilde{A}=$ $A / D, \tilde{B}=B / D$ are $\langle\tilde{h}\rangle$-conjugacy separable. Thus $A, B$ are $H$-conjugacy separable. Moreover, they are double coset separable [9], and hence $A, B$ are $\left\langle h^{n}\right\rangle$ double coset separable for each $n>0$. Since $G / D$ is a generalized free product of finitely generated nilpotent groups amalgamating a cyclic subgroup, $G / D$ is conjugacy separable [4]. By Lemma 5.2, $(C)$ holds for $A, B$. Since finitely generated nilpotent groups are $\langle h\rangle$-self-conjugate, $G$ is conjugacy separable by Theorem 4.5 .

Theorem 5.4. Let $G=A *_{H} B$ with $H=\langle h\rangle \times D$, where $D \subset Z(A) \cap Z(B)$. If $A, B$ are finitely generated nilpotent, then $G$ is conjugacy separable.

Proof. Let $x, y \in G$ be elements of minimal lengths in their conjugate classes and $x \psi_{G} y$. Since $\tilde{G}=G / D=\tilde{A} *_{\langle\tilde{h}\rangle} \tilde{B}$ is conjugacy separable by [4], if $\tilde{x} \chi_{\tilde{G}} \tilde{y}$, then we can find $\tilde{P} \triangleleft_{f} \tilde{G}$ such that $\tilde{P} \tilde{x} \chi_{\tilde{G} / \tilde{P}} \tilde{P} \tilde{y}$. Let $P$ be the preimage of $\tilde{P}$. Then $P \triangleleft_{f} G$ and $P x \chi_{G / P} P y$. Hence we assume that $\tilde{x} \sim_{\tilde{G}} \tilde{y}$. Then $y \sim_{G} x u$ for some $u \in D$. Hence we can take $y=x u$ and $x \varkappa_{G} x u$, where $u \in D$. Let $D_{1}=\left\{c \in D \mid x \sim_{G} x c\right\}$. Then $D_{1}$ is a subgroup of $D$. Clearly, $u \notin D_{1}$. Since $D$ is finitely generated abelian, there exists $D_{2} \triangleleft_{f} D$ such that $u \notin D_{2} D_{1}$. Let $\bar{G}=G / D_{2}$. Then $\bar{G}=\bar{A} * \bar{H} \bar{B}$, where $\bar{H}=\langle\bar{h}\rangle \times \bar{D}$. It is easy to see that $\bar{x} \chi_{\bar{G}} \overline{x u}$. Clearly, $|h|=|\bar{h}|$. Since $\bar{D}$ is finite, if $\langle\bar{h}\rangle$ is finite, then $\bar{H}$ is finite and $\bar{G}$ is conjugacy separable by [4]. On the other hand, if $\langle\bar{h}\rangle$ is infinite, then $\bar{G}$ is conjugacy separable by Theorem 5.3 . Thus there exists $\bar{P} \triangleleft_{f} \bar{G}$ such that $\bar{P} \bar{x} \chi_{\bar{G}} / \bar{P} \bar{P} \overline{x u}$. Let $P$ be the preimage of $\bar{P}$. Then $P \triangleleft_{f} G$ and $P x \chi_{G / P} P y$. Hence $G$ is conjugacy separable. 
Note that free groups satisfy most of the conditions in Theorem 4.5 except that they only have trivial centers. Thus we can apply Theorem 4.5 to show conjugacy separability of the following example:

Example 5.5. Let $G=G_{1} *_{H} G_{2}$, where $G_{i}=F_{i} \times S_{i}(i=1,2)$, with $F_{i}$ free and $S_{i}$ finitely generated nilpotent groups and $H=\langle h\rangle \times D$, where $\langle h\rangle=F_{1} \cap F_{2}$ and $D \subset Z\left(S_{1}\right) \cap Z\left(S_{2}\right)$. Then $G$ is conjugacy separable.

Proof. It is well-known that free groups $F_{i}$ are conjugacy separable, $\langle h\rangle$-selfconjugate, and $\left\langle h^{n}\right\rangle$-double coset separable for each $n>0$. Hence $G_{i}=F_{i} \times S_{i}$ are also conjugacy separable, $\langle h\rangle$-self-conjugate, and $\left\langle h^{n}\right\rangle$-double coset separable for each $n>0$. It is also well-known that free groups $F_{i}$ satisfy $\left(C^{\prime}\right)$ in Theorem 3.5. Hence $G_{i}$ satisfy $(C)$. Note that $G_{i} / D \cong F_{i} \times \bar{S}_{i}$, where $\bar{S}_{i}=S_{i} / D$ is finitely generated nilpotent. Every finitely generated nilpotent group is a finite extension of a finitely generated torsion-free nilpotent group. Hence each $\bar{S}_{i}$ is a finite extension of a finitely generated torsion-free nilpotent group $\bar{T}_{i}$. Then $G_{i} / D$ is isomorphic to a finite extension of $F_{i} \times \bar{T}_{i}$. We note that the group $F_{i} \times \bar{T}_{i}$ is a residually finitely generated torsion-free nilpotent group. Hence $G / D=G_{1} / D *\langle\bar{h}\rangle G_{2} / D$ is conjugacy separable [7]. Since free groups are cyclic conjugacy separable, $G_{i} / D \cong F_{i} \times \bar{S}_{i}$ are $\langle h\rangle$-conjugacy separable. Hence each $G_{i}$ is $H$-conjugacy separable. By Theorem $4.5, G$ is conjugacy separable when $D$ is finite. Then as in the proof of Theorem 5.4, we can show that $G$ is conjugacy separable when $D$ is infinite.

Acknowledgements. We would like to thank the anonymous referee for his or her generous advice.

\section{References}

[1] G. Baumslag, A non-Hopfian group, Bull. Amer. Math. Soc. 68 (1962), 196-198.

[2] N. Blackburn, Conjugacy in nilpotent groups, Proc. Amer. Math. Soc. 16 (1965), 143148.

[3] J. L. Dyer, Separating conjugates in free-by-finite groups, J. London Math. Soc. (2) 20 (1979), no. 2, 215-221.

[4] Separating conjugates in amalgamated free products and HNN extensions, J. Austral. Math. Soc. Ser. A 29 (1980), no. 1, 35-51.

[5] B. Fine and G. Rosenberger, Conjugacy separability of Fuchsian groups and related questions, Combinatorial group theory (College Park, MD, 1988), 11-18, Contemp. Math., 109, Amer. Math. Soc., Providence, RI, 1990.

[6] E. Formanek, Conjugate separability in polycyclic groups, J. Algebra 42 (1976), no. 1, 1-10.

[7] G. Kim and C. Y. Tang, Conjugacy separability of generalized free products of finite extensions of residually nilpotent groups, In Group Theory (Proc. of the '96 Beijing Int'l Symposium), pages 10-24. Springer-Verlag, 1998.

[8] - Separability properties of certain tree products of groups, J. Algebra 251 (2002), no. 1, 323-349.

[9] John C. Lennox and John S. Wilson, On products of subgroups in polycyclic groups, Arch. Math. 33 (1979), no. 4, 305-309. 
[10] W. Magnus, A. Karrass, and D. Solitar, Combinatorial Group Theory, Pure and Applied Math. Vol. XIII, Wiley-Interscience, New York-London-Sydney, 1966.

[11] A. I. Mal'cev, Homomorphisms of finite groups, Ivanov Gos. Ped. Inst. Učen. Zap. Uchen. Zap. Karel. Ped. Inst. Ser. Fiz.-Mat. Nauk 18 (1958), 49-60.

[12] A. W. Mostowski, On the decidability of some problems in special classes of groups, Fund. Math. 59 (1966), 123-135.

[13] G. A. Niblo, Separability properties of free groups and surface groups, J. Pure and Appl. Algebra 78 (1992), no. 1, 77-84.

[14] V. N. Remeslennikov, Conjugacy in polycyclic groups, Algebra Log. 8 (1969), 712-725.

[15] _ Groups that are residually finite with respect to conjugacy, Siberian Math. J. 12 (1971), 783-792.

[16] L. Ribes, D. Segal, and P. A. Zalesskii, Conjugacy separability and free products of groups with cyclic amalgamation, J. London Math. Soc. 57 (1998), no. 3, 609-628.

[17] L. Ribes and P. A. Zalesskii, On the profinite topology on a free group, Bull. London Math. Soc. 25 (1993), no. 1, 37-43.

[18] P. Scott, Subgroups of surface groups are almost geometric, J. London Math. Soc. 17 (1978), no. 3, 555-565.

[19] P. F. Stebe, A residual property of certain groups, Proc. Amer. Math. Soc. 26 (1970), $37-42$.

[20] C. Y. Tang, Conjugacy separability of generalized free products of surface groups, J. Pure Appl. Algebra 120 (1997), no. 2, 187-194.

[21] J. S. Wilson and P. A. Zalesskii, Conjugacy separability of certain Bianchi groups and HNN extensions, Math. Proc. Cambridge Philos. Soc. 123 (1998), no. 2, 227-242.

GoANSU Kim

Department of Mathematics

YeUnGNAM UNIVERSITY

KYONGSAN, 712-749, KoREA

E-mail address: gskim@yu.ac.kr

C. Y. TANG

Department of Mathematics

UNIVERSITY OF WATERLOO

Waterloo, Ontario, N2L 3G1, Canada

E-mail address: fcytang@math.uwaterloo.ca 